\title{
Evaluation of neuropathic pain occurring after high- dose-rate interstitial brachytherapy of oral tongue
}

\author{
Vijai Simha, MD, Suresh C. Sharma, MD, Rakesh Kapoor, MD, Chirag K. Ahuja, MD, Arun S. Oinam, PhD, \\ Sushmita Ghoshal, MD \\ Department of Radiation Oncology, PGIMER, Chandigarh, India
}

\begin{abstract}
Purpose: To recognize neuropathic pain as a complication of high-dose-rate (HDR) interstitial brachytherapy of oral tongue and to evaluate the possible causes of neuropathy.

Material and methods: Twenty one patients who underwent interstitial brachytherapy for early cancer of oral tongue were evaluated. The patients either underwent primary brachytherapy (42-48 Gy at 3-4 Gy/fraction) or a boost (18-24 Gy at $3 \mathrm{~Gy} /$ fraction) after external radiation to $40 \mathrm{~Gy}$. Lingual nerve was the nerve concerned and the sublingual space (SLS) was contoured as its surrogate. Dosimetric parameters were correlated with onset of pain.

Results: Ten patients out of 21 (47.61\%) developed painful neuropathy. Five patients of six (5/6) who underwent primary brachytherapy developed neuropathy. Five out of $15(5 / 15)$ patients who underwent brachytherapy as a boost developed neuropathy. The patients who underwent primary brachytherapy were ten times more likely to develop neuropathy. Among the patients receiving boost treatment, the equivalent dose at $2 \mathrm{~Gy} /$ fraction (EQD2) to 2 cc of SLS was higher (39.25 Gy) in the patients who developed pain compared to those without pain (10.29 Gy).

Conclusions: This is the first report to recognize neuropathic pain as a complication of HDR brachytherapy of oral tongue. Patients undergoing primary brachytherapy were more likely to develop pain. Among other factors like dose to SLS, number of catheters, size of the primary tumor, and the dose rate, only dose to 2 cc of the SLS correlated with onset of pain. The SLS (containing the lingual nerve) may be considered an organ at risk to prevent the occurrence of this complication.

J Contemp Brachytherapy 2015; 7, 2: 142-146

DOI: $10.5114 /$ jcb.2015.50658
\end{abstract}

Key words: high-dose-rate brachytherapy, interstitial brachytherapy, neuropathy, oral tongue, painful neuropathy.

\section{Purpose}

Localized cancer of the tongue is an ideal indication for brachytherapy [1,2] because emphasis is laid mainly on local control while maintaining anatomical integrity and function. Though surgery is considered the standard of care, significant functional impairment may result after wide local excision of even the smallest tumors. Radiotherapy has produced results comparable to surgery and is considered an acceptable alternative. Eighty five percent of the tumors involving the anterior two thirds of the tongue arise from the lateral border of the oral tongue. These are detected at an early stage as they become symptomatic early, and are amenable to clinical examination and accessible to interstitial implant. In our practice, a complication often seen after interstitial implant of the tongue is development of painful neuropathy. The pain is perceived mainly at the local site and often radiates to the area of distribution of the ipsilateral trigeminal nerve. The pain develops after a successful outcome when there is no residual disease on clinical examination or imaging, which is quite distressing to the patient as well as the phy- sician. The nerve concerned is the lingual nerve (branch of mandibular division of the trigeminal nerve $V_{3}$ ), which carries general sensation including pain from the anterior $2 / 3^{\text {rd }}$ of the tongue. This nerve lies in the sublingual space (SLS) inferolateral to the root of the tongue where it could be damaged during brachytherapy [3,4] (Fig. 1) or get compressed due to massive edema of the tongue in some cases. Using high-dose-rate (HDR) brachytherapy, the reported local control rates range from $53-100 \%$ [5-7]. Leung et al. [7] reported local control rate of $94.7 \%$ for early stage and only acute mucositis as a complication after HDR brachytherapy. Bhalavat et al. [8], based on low-dose-rate (LDR) brachytherapy, have reported local control of $59.7 \%$. The two common complications reported by them was soft tissue necrosis in $12.5 \%$ and osteoradionecrosis in $3.5 \%$.

So far in the literature, no study has reported development of neuropathy after interstitial implant of oral tongue. In this study, we try to evaluate the cause of the pain, which persists in spite of excellent local control of the disease in the tongue. 


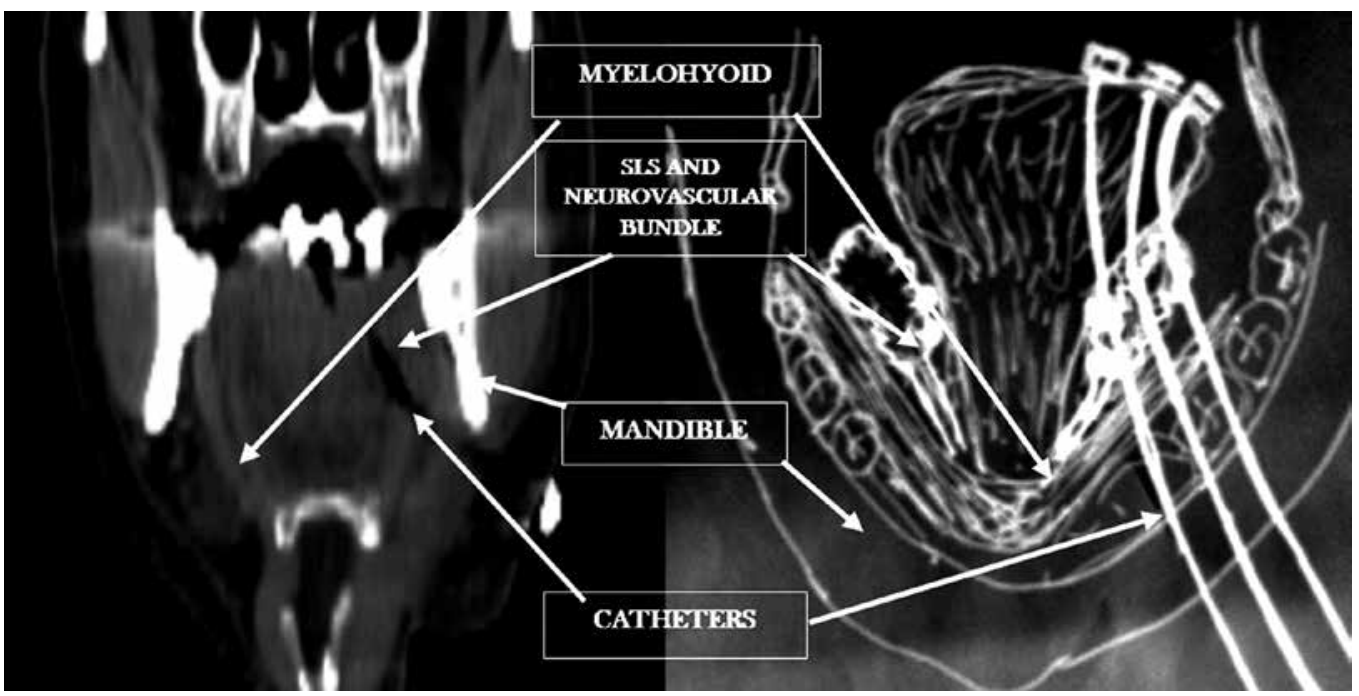

Fig. 1. Diagram showing how the lingual nerve can be injured when catheters are inserted through the sublingual via submandibular space. Computed tomography is shown on the left and a corresponding schematic diagram on the right. SLS - sublingual space

\section{Material and methods}

Twenty one patients who underwent interstitial brachytherapy for the mobile part of the tongue, either as a primary treatment or as a boost after external beam radiation therapy (EBRT) were considered. They were treated in our institute between November 2009 and August 2013. Only T1 and T2 tumors (American Joint Committee on Cancer, AJCC stage I and II) of the oral tongue situated on the lateral border were included in this study. After biopsy confirmation of malignancy, all patients underwent thorough clinical examination and computed tomography (CT) of the neck for staging of nodal disease. Only clinically node negative $\left(\mathrm{N}_{0}\right)$ patients were considered for brachytherapy. The patients either underwent EBRT to dose of $40 \mathrm{~Gy} / 20 \#$ followed by brachytherapy boost or were taken up for primary brachytherapy, if found suitable at the time of initial diagnosis. During brachytherapy, implantation was done under general anesthesia by the "push and pull technique" with the needle inserted below the jaw and medial to the mandible (Fig. 1) and by retrograde pulling plastic loops through the needle. Implantation was done following the rules of the Paris system [9], in single or double plane, giving 5-10 $\mathrm{mm}$ margin from all clinically palpable disease. Additional buttons were used to improve the dose to the surface of the tongue. A spacer was not used between the tongue and the mandible. Subsequent to implantation, the patients underwent a non-contrast CT scan for treatment planning. The CT images were acquired on GE LightSpeed CT Scanner (GE Healthcare, Chalfont St. Giles, UK, a unit of General Electric Company) with slice thickness of $2.5 \mathrm{~mm}$. Treatment planning was done on the Oncentra Master Plan ${ }^{\mathrm{TM}}$ v3.0 software Nucletron, an Elekta company (Elekta AB, Stockholm, Sweden) where catheters were subsequently reconstructed and dose was prescribed to a point $5 \mathrm{~mm}$ outside the plane of the implant. The length of loading of the catheters, which would determine the final volume of the implant was left to clinician's discretion. Subsequently, they were treated to a dose of 42-48 Gy at 3-4 Gy/fraction for primary brachytherapy treatment and a dose of 18-24 Gy at $3 \mathrm{~Gy} /$ fraction when brachytherapy was used as a boost after EBRT. Two fractions per day were delivered with a minimum gap of six hours between the fractions. Treatment was delivered with Iridium $\left(\operatorname{Ir}^{192}\right)$ using a remote controlled after-loading machine (Nucletron, an Elekta company). Retrospectively, contouring of the sublingual space (SLS) was done, which was used as a surrogate for the lingual nerve. Sublingual space was contoured as the low density fat containing space below the root of the tongue between the geniohyoid medially and the myelohyoid muscles laterally (Fig. 2).

The case records of the patients were reviewed to determine the onset and nature of pain. The date of the first occurrence of neuropathic pain after a minimum of six weeks after completion brachytherapy in the absence of palpable disease/local ulceration/soft tissue necrosis/ osteoradionecrosis was considered as a primary end point of the study. The patients were followed-up with regular two-monthly clinical visits. The last follow-up visit or development of local recurrence was considered a secondary endpoint of this study. The dose to the SLS was obtained as $\mathrm{D}_{2 \mathrm{cc}}$ (minimum dose to maximally exposed $2 \mathrm{cc}$ volume of SLS) from the dose volume histogram of the Oncentra Master Plan ${ }^{\mathrm{TM}}$ v3.0 software (Nucletron, an Elekta company). The cumulative dose to the lingual nerve was calculated assuming $\alpha / \beta$ ratio of 3 for the SLS. The total dose was calculated from the linear quadratic model and expressed as EQD2 for the SLS. Statistical analysis was done using the Excel 2007 software package, and independent $t$-tests and Analysis of variance (ANOVA) were applied to find the differences between the means of those who developed pain and those who did not develop pain.

\section{Results}

Twenty one patients were evaluated (Table 1), out of which $6(28.57 \%)$ underwent upfront brachytherapy. Fifteen $(71.4 \%)$ underwent EBRT to dose of 40 Gy in 20 frac- 


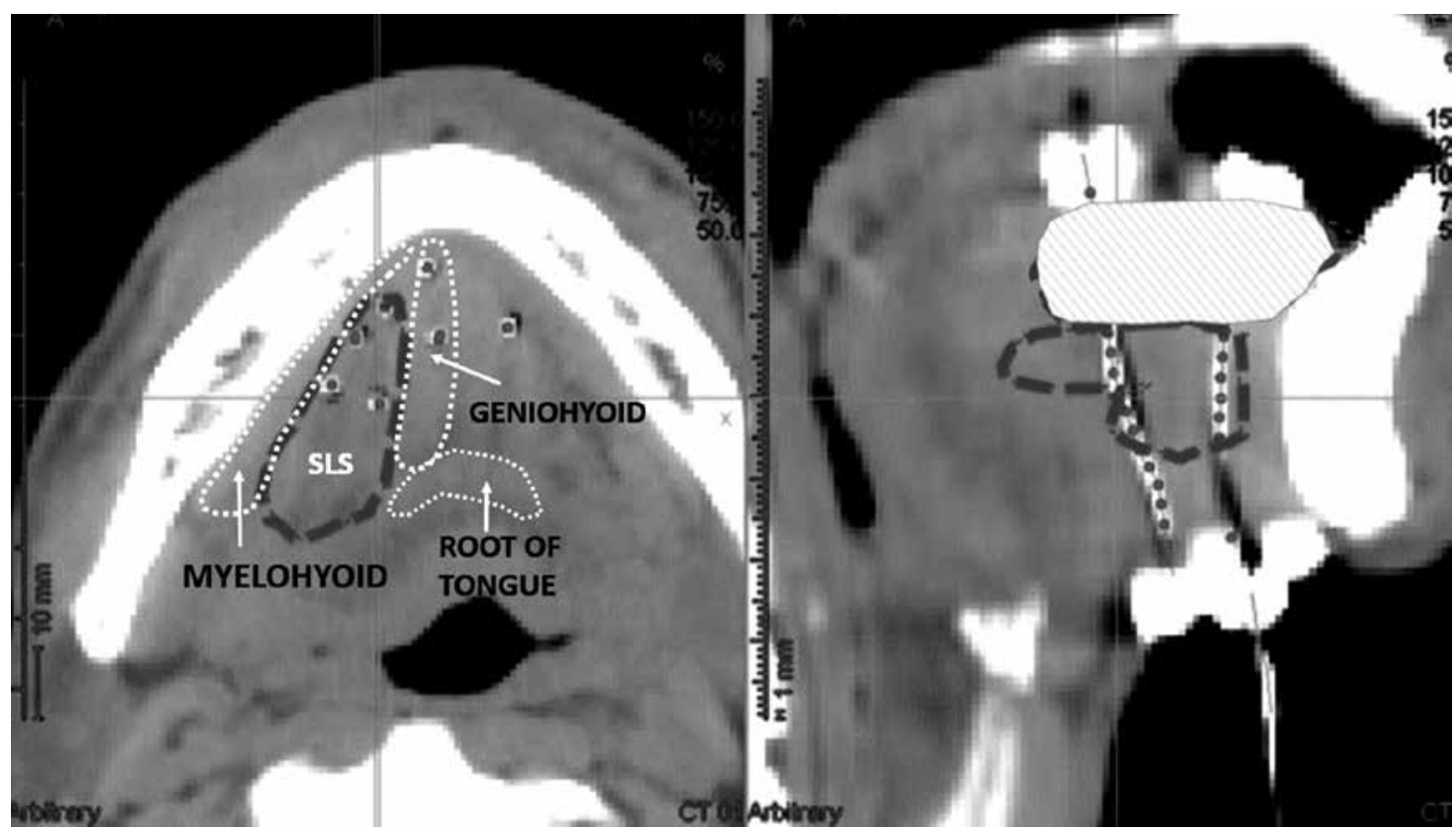

Fig. 2. Multi-planar reconstructed view showing the contoured sublingual space (SLS) below the root of the tongue between the geniohyoid and the myelohyoid muscles. The full thickness of the dorsum of the tongue is represented by the shaded structure

tions followed by brachytherapy implant as a boost. Median follow up period was 12 months (4-38 months). Ten (47.6\%) patients had onset of neuropathic pain. The average time for onset of neuropathic pain was 4.23 months (1.8-7.2 months) from completion of brachytherapy. Five out of six $(83 \%)$ patients who had received primary brachytherapy developed painful neuropathy. Five out of 15 patients (33\%) who received brachytherapy as boost after EBRT developed neuropathy. Using the odds ratio (Table 2), the probability of developing neuropathy was 10 times higher when patients underwent primary brachytherapy implant rather than an implant as a boost after EBRT.

We could not compare the dose to SLS of patients who underwent primary brachytherapy with those who

Table 1. The age, sex, and clinical stage of the patients included in the study

\begin{tabular}{ll} 
Factor & Number $(n=21)$ \\
\hline Age & \\
\hline $20-40$ & $6(28.57 \%)$ \\
\hline $40-60$ & $9(42.85 \%)$ \\
\hline $60-80$ & $6(28.57 \%)$ \\
\hline Sex & $15(71.42 \%)$ \\
\hline Males & $6(28.57 \%)$ \\
\hline Females & $9(42.85 \%)$ \\
\hline Stage & $12(57.14 \%)$
\end{tabular}

received brachytherapy as a boost because the dose prescription at the time of brachytherapy was different. Also, the overall treatment time was different between the two groups (patients undergoing primary brachytherapy received larger dose in a shorter period of time). Hence, they are described as two different groups. The average dose to $2 \mathrm{cc}\left(\mathrm{D}_{2 \mathrm{cc}}\right)$ contoured SLS in the patients who underwent primary brachytherapy was $56.49 \pm 21.52$ Gy (23.6-77.8 Gy) EQD2. Among patients undergoing brachytherapy as a boost the average $\mathrm{D}_{2 c c}$ to the SLS was $19.94 \pm$ 19.36 Gy (1.81-64.41 Gy) EQD2. Of the 15 patients who underwent brachytherapy as a boost, five patients $(33.3 \%)$ developed neuropathic pain. In this group, the average total $\mathrm{D}_{2 \mathrm{cc}}$ to SLS was higher $-39.25 \pm 18.74 \mathrm{~Gy}$ (21.74-64.4 Gy) EQD2 compared to patients who did not develop pain $10.29 \pm 6.08$ Gy (1.81-16.74 Gy) EQD2. Independent $t$-test was used to compare the difference and was statistically significant $(p=0.04)$. ANOVA test applied for depth of prescription of the implant was not a statistically significant factor in determining the onset of neuropathic pain $(p=0.11)$. Also, the ANOVA for the number of needles implanted or the dose rate between the two groups was not statistically significant $(p=0.16$ and 0.77 ) respectively (Table 3).

Table 2. The causative relation between upfront brachytherapy and occurrence of neuropathic pain

\begin{tabular}{lcc} 
& $\begin{array}{c}\text { Onset of pain } \\
10(47.61 \%) \\
\text { patients }\end{array}$ & $\begin{array}{c}\text { No onset of pain } \\
11(52.38 \%) \\
\text { patients }\end{array}$ \\
\hline Primary brachytherapy & 5 & 1 \\
\hline Brachytherapy boost & 5 & 10
\end{tabular}


Table 3. The dosimetric parameters in those who underwent brachytherapy as a boost

\begin{tabular}{lcc} 
& $\begin{array}{c}\text { Onset of neuropathic pain } \\
\text { (5 patients) }\end{array}$ & $\begin{array}{c}\text { No onset of neuropathic pain } \\
\text { (10 patients) }\end{array}$ \\
\hline Average dose EQD2 to the SLS 2 cc in the interstitial boost group & $39.25 \pm 18.74 \mathrm{~Gy}$ & $10.29 \mathrm{~Gy}$ \\
\hline Average depth of loading & $40 \mathrm{~mm}(15-40 \mathrm{~mm})$ & $40 \mathrm{~mm}(18-44 \mathrm{~mm})$ \\
\hline Loading of SLS (source dwell position within SLS) & 6 patients & 4 patients \\
\cline { 2 - 3 } Dose rate & $1.82 \pm 1.02 \mathrm{~Gy} / \mathrm{min}$ & $2.01 \pm 1.68 \mathrm{~Gy} / \mathrm{min}$ \\
EQD2 - equivalent dose at 2 Gy per fraction, SLS - sublingual space, Gy - Gray & &
\end{tabular}

In seven of the ten patients who developed neuropathic pain, the pain persisted up to the last follow-up date. Three patients had resolution of the pain after an average period of fifteen months (7-22 months). Overall, three patients developed local recurrence and two had nodal recurrence. These patients were referred for salvage surgery. Among those who developed local recurrence, only one of the patient had neuropathic pain, which persisted till the date of documented local recurrence. In this patient, the neuropathic pain predated the recurrence by 11 months. After a median follow up period of 12 months (4-38 months), 16 (76.19\%) patients were locally disease free.

\section{Discussion}

The injury to the lingual nerve during oro-dental procedures is a well-recognized complication. The injury usually occurs during the extraction of the mandibular $3^{\text {rd }}$ molar tooth where the lingual nerve runs in a close relation to the dental alveolus [10-12]. The complications after interstitial brachytherapy reported so far apart from acute mucositis have been soft tissue necrosis and osteoradionecrosis. Though the neuropathic pain has not been reported in literature on interstitial brachytherapy, we are seeing it in our practice $(47.6 \%$ incidence in this study) due to the use of higher dose rates [13-15] and use of techniques where the needles traverse the sublingual space, which contains the terminal part of the lingual nerve as it ramifies to supply general sensation to the anterior $2 / 3^{\text {rds }}$ of the tongue. As far as local control is concerned, fractionated HDR interstitial brachytherapy has been shown to be an effective alternative to traditional LDR brachytherapy for early tongue cancer $[5,16]$. However, previous studies have cautioned regarding use of higher doses and dose rates, which was correlated with increased incidence of complications particularly necrosis [14-17]. It is generally accepted that the experience is much less with HDR and long time is needed to get the clinical and scientific experience that has been accumulated for decades with the use of LDR technique [18]. High activity sources create dose-rate conditions that are different from LDR, making necessary new, more precise radiobiological tools for schedule inter-comparison [19]. In this study, we observed that patients who underwent primary brachytherapy had a 10 times higher risk of developing neuropathic pain, which suggests that the hypo-fractionated dose delivered at a higher dose rate injuring the nerve is likely to be the main culprit. As CT scan cannot clearly identify the lingual nerve in this region, we have used the sublingual space, which is seen easily on CT scans as low density fat containing space as a surrogate for the lingual nerve [4]. Though the mere placement of a needle through the sublingual space could result in injury to the lingual nerve, the presence of a lag period of an average of 4.23 months for the onset of the pain suggests radiation induced damage to the lingual nerve as the more probable cause (Fig. 3). The other factors that can be held responsible for the causation of pain are the development of edema in the tongue after radiation causing compression of the nerve similar to the 'entrapment neuropathies' seen in the extremities.

Similar factors that can potentially increase the dose to the SLS have been evaluated; namely, the length of loading of the catheters, number of catheters implanted, and dose rate. These factors did not significantly correlate with the onset of pain. The only factor associated to the onset of pain apart from the use of HDR brachytherapy was the increased total dose (EQD2) $\mathrm{D}_{2 \mathrm{cc}}$ of the SLS, which would primarily be determined by the depth of the implant. In the era of CT/MRI based planning, the depth can be reasonably restricted and at the same time the tumor can be adequately irradiated. The data on the damage to the peripheral nerves from HDR radiation comes from mainly with the use of intraoperative radiotherapy (IOERT) where peripheral nerve is the principal dose-limiting normal tissue for IOERT in the pelvis, ret-

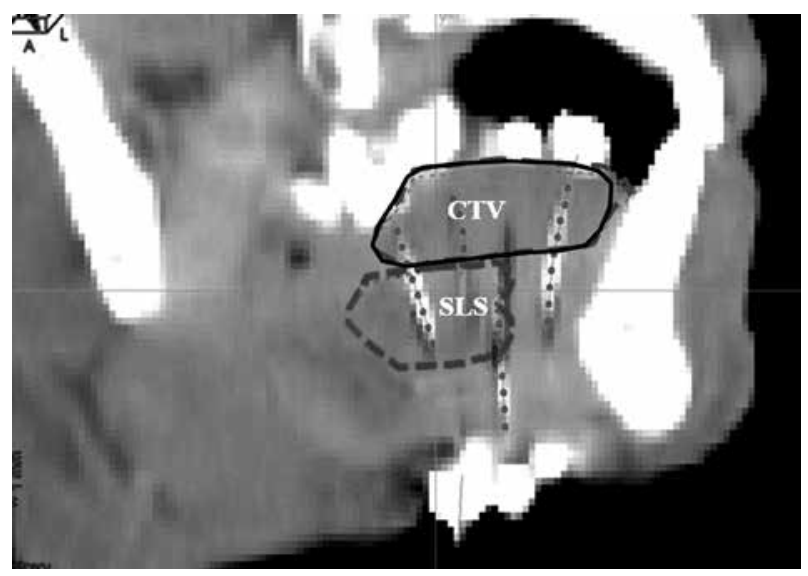

Fig. 3. Showing how excess loading of the catheters to the depth may result in overdosing of sublingual space (SLS) and beyond by multiple catheters and leading to lingual nerve injury 
roperitoneum, and extremities $[13,20]$. Neuropathy was observed in $65 \%$ of the patients when they received doses of $>15 \mathrm{~Gy}$ and the onset of neuropathy was related to the length of the nerve irradiated [13].

The neuropathic pain resolved spontaneously in three $(30 \%)$ patients and persisted to the last follow up date in the remaining seven suggesting that over a period of time the pain may resolve by itself. However, this is a retrospective analysis and we are not able to describe the impact of pain on the quality of life. Though, a correlation between onset of pain and $D_{2 c c}$ to SLS has been established in this study, definite correlation between the severity of the pain, requirement of analgesics, and the dose to SLS cannot be arrived at. Prospective studies are required with pain and quality of life questionnaires to know the impact of irradiated volume on occurrence of neuropathy as well as the quality of life. Also, the incidence of neuropathic pain may not be the true incidence of this complication as we were not able to objectively confirm presence of nerve damage. At the best excess irradiation of the SLS (determined primarily by the depth of the treatment volume) with high dose radiation, may be used as an indicator of development of neuropathic pain. Also, it is important to realize that there are uncertainties due to development of edema when the implant is kept in-situ for a period of 3-4 days, particularly if the treatment volume and dose to SLS is decided on the basis of the CT scan taken on the day of the implant.

Considering the secondary endpoints of this study, $76.19 \%$ of the patients were disease free till the last clinical visit, which is comparable to what has been reported in literature [5-7]. Out of the 10 patients who developed neuropathic pain, only one had local recurrence, which predated local recurrence by 11 months. This suggests that the neuropathic pain was unlikely due to a smoldering residual disease.

\section{Conclusions}

From this article, we would like to recognize neuropathic pain as a complication of modern HDR interstitial implant brachytherapy of the tongue and radiation induced injury to the lingual nerve as the most likely cause of neuropathy. The incidence of neuropathic pain was 10 times more when brachytherapy was used as a sole modality. In the patients who developed pain after brachytherapy for boost, the dose to 2 cc of the SLS correlated with the onset of pain. Use of sectional imaging like CT scan and an MRI at the time of brachytherapy will help to identify sublingual space, which may be considered as an organ at risk (OAR) to prevent overdosing of the lingual nerve. Also, the use of MRI or CT scan at the time of brachytherapy helps to define the CTV, to which the dose may be evaluated and the depth of the treatment volume adequately restricted. Hence, a large dose to smaller area of the tongue may be delivered increasing the therapeutic efficacy of brachytherapy.

\section{Disclosure}

Authors report no conflict of interest.

\section{References}

1. Mazeron JJ, Ardiet JM, Haie-Meder C et al. GEC-ESTRO recommendations for brachytherapy for head and neck squamous cell carcinomas. Radiother Oncol 2009; 91: 150-156.

2. Yamazaki H, Yoshida K, Yoshioka $Y$ et al. High dose rate brachytherapy for oral cancer. J Radiat Res 2013; 54: 1-17.

3. Gray H, Pickering PT, Howden R. Gray's Anatomy, descriptive and surgical. Bounty Books, New York 1977; p. 485.

4. Arya S, Chaukar D, Pai P. Imaging in oral cancers. Indian J Radiol Imaging 2012; 22: 195-208.

5. Inoue T, Inoue T, Teshima T et al. Phase III trial of high and low dose rate interstitial radiotherapy for early oral tongue cancer. Int J Radiat Oncol Biol Phys 1996; 36: 1201-1204.

6. Lau HY, Hay JH, Flores AD et al. Seven fractions of twice daily high dose-rate brachytherapy for node-negative carcinoma of the mobile tongue results in loss of therapeutic ratio. Radiother Oncol 1996; 39: 15-18.

7. Leung TW, Wong VY, Kwan $\mathrm{KH}$ et al. High dose rate brachytherapy for early stage oral tongue cancer. Head Neck 2002; 24: 274-281.

8. Bhalavat RI, Mahantshetty UM, Tole S et al. Treatment outcome with low-dose-rate interstitial brachytherapy in early-stage oral tongue cancers. J Cancer Res Ther 2009; 5: 192197.

9. Pierquin B, Dutreix A, Paine $\mathrm{CH}$ et al. The Paris system in interstitial radiation therapy. Acta Radiol Oncol Radiat Phys Biol 1978; 17: 33-48.

10. Fielding AF, Rachiele DP, Frazier G. Lingual nerve paresthesia following third molar surgery: a retrospective clinical study. Oral Surg Oral Med Oral Pathol Oral Radiol Endod 1997; 84: 345-348.

11. Malden NJ, Maidment YG. Lingual nerve injury subsequent to wisdom teeth removal - a 5-year retrospective audit from a high street dental practice. Br Dent J 2002; 193: 203-205.

12. McGeachie JK. Anatomy of the lingual nerve in relation to possible damage during clinical procedures. Ann $R$ Australas Coll Dent Surg 2002; 16: 109-110.

13. Gunderson LL. Intraoperative irradiation: techniques and results. Humana Press, Totowa 1999, pp. 126-128.

14. Mazeron JJ, Simon JM, Le Pechoux C et al. Effect of dose rate on local control and complications in definitive irradiation of T1-2 squamous cell carcinomas of mobile tongue and floor of mouth with interstitial iridium-192. Radiother Oncol 1991; 21: $39-47$.

15. Nag S, Cano ER, Demanes DJ et al. The American Brachytherapy Society recommendations for high-dose-rate brachytherapy for head-and-neck carcinoma. Int J Radiat Oncol Biol Phys 2001; 50: 1190-1198.

16. Yamazaki $H$, Inoue $T$, Yoshida $K$ et al. Brachytherapy for early oral tongue cancer: low dose rate to high dose rate. J Radiat Res 2003; 44: 37-40.

17. Petera J, Matula P, Paluska P et al. High dose rate versus low dose rate brachytherapy in the treatment of tongue carcinoma - a radiobiological study. Neoplasma 2009; 56: 163-168.

18. Fijuth J. Is there any place for LDR brachytherapy for head and neck carcinomas in HDR era? J Contemp Brachytherapy 2009; 1: 62-66.

19. Polo A. High-dose-rate and pulsed-dose-rate brachytherapy for oral cavity cancer and oropharynx cancer. J Contemp Brachytherapy 2009; 1: 216-223.

20. McMahon SB, Priestley JV. Peripheral neuropathies and neurotrophic factors: animal models and clinical perspectives. Curr Opin Neurobiol 1995; 5: 616-624. 